\title{
On the shuttle glow (the plasma alternative)
}

\author{
K. Papadopoulos ${ }^{1}$ \\ Science Applications, Inc.
}

(Received April 8, 1983; revised September 14, 1983; accepted October 12, 1983.)

\begin{abstract}
It is shown that the anomalous glow observed in the recent space shuttle flights can be attributed to a combination of beam plasma discharge and critical ionization phenomena. While the shuttle velocity $\left(\sim 8 \mathrm{~km} \mathrm{~s}^{-1}\right)$ is below the critical ionization velocity, which for oxygen is $12.7 \mathrm{~km} \mathrm{~s}^{-1}$, specular reflection of a small fraction (2-3\%) of ambient ions forms an ion beam with velocity $16 \mathrm{~km} \mathrm{~s}^{-1}$, making up for the energy deficit in the critical ionization velocity phenomenon. The wave activity observed is consistent with this model.
\end{abstract}

The recently released photographs taken during the third and fourth flights of the space shuttle revealed a significant illumination of the surfaces of the vehicle facing the direction of the velocity vector [Banks et al., 1983; Mende et al., 1983]. These observations, besides presenting a significant scientific puzzle, are of major practical significance to future astronomical and geophysical payloads, because of the optical and IR contamination that they cause in the orbiter vicinity. All attempted interpretations [Yee and Abreu, 1983; Slanger, 1983] of the shuttle glow, as well as of the glow observed on the Atmosphere Explorer satellites [Torr, 1983], have concentrated on purely chemical effects, related to molecular species excited by the interaction of the moving shuttle surfaces with the ambient neutral gas. Although the surface interactions of the neutral gas are quite important in determining the equilibrium or dynamic composition of the atomic and molecular species near the shuttle, and the emission from metastable or recombining processes, they are only a part of the total interaction. The plasma diagnostics package (PDP) measurements [Shawhan and Murphy, 1983] on STS 3 have shown a very active plasma environment, whose role in the optical and IR emissions has been generally ignored. Simply attempting to establish the spectral character of the emission, while ignoring the character of the associated plasma

\footnotetext{
${ }^{1}$ Permanently at University of Maryland.
}

Copyright 1984 by the American Geophysical Union.

Paper number 3S1677.

0048-6604/84/003S-1677\$08.00 environment, might produce a very ambiguous and subjective interpretation, which by no means can be used to evaluate the impact of the spacecraft-related contamination on future shuttle payloads or space platforms and telescopes. The following summary of the plasma environment measured by the PDP convincingly indicates that collective plasma processes play a large role in the proper evaluation of the orbiter contamination:

1. The plasma density $n$ in the vicinity of the orbiter was often found to be several times and up to an order or magnitude higher than the ambient plasma density $n_{0}$. It is like an ionized cloud surrounding selective areas of the spacecraft.

2. Energetic $(20-100 \mathrm{eV})$ electron fluxes up to $10^{14} \mathrm{el} \mathrm{cm}^{-2} \mathrm{~s}^{-1}$ were measured, the maximum occurring during daytime conditions.

3. Ion fluxes with energies of up to $30 \mathrm{eV}$ were observed, sometimes coincident with single or counterstreaming ion beams with $10-\mathrm{eV}$ mean energy.

4. In addition to $\mathrm{O}^{+}, \mathrm{NO}^{+}$, and $\mathrm{O}_{2}^{+}, \mathrm{H}_{2} \mathrm{O}^{+}$was observed, being on occasion the dominant species.

5. An intense electrostatic broadband noise between low- and high-frequency cutoffs at $30 \mathrm{~Hz}$ and $20 \mathrm{kHz}$, respectively, was measured, with amplitude up to $1 \mathrm{~V} \mathrm{~m}^{-1}$.

6. A gas layer of up to $10^{-5}$ torr was associated with surfaces ramming into atmospheric gases. Large pressure increases were observed during thruster operations and with payload bay doors closed.

7. Most of the plasma as well as the glow phenomena were intensified during daytime ram conditions and during thruster operations.

The above observations, in conjunction with the 
optical emissions, pose several important scientific questions connected with understanding the environment of the space shuttle and space platforms and the related scalings, such as the following:

1. What causes the increase in the ambient plasma density in the orbiter vicinity?

2. What is the source of the energization of electrons to $100 \mathrm{eV}$ and of ions to $30 \mathrm{eV}$ ? Notice that all laminar potentials are below $5 \mathrm{eV}$, indicating the need for turbulent acceleration.

3. What is the role of the ionizing processes and the energetic electron fluxes in the optical and IR emissions?

4. How is the equilibrium or dynamic composition of the neutral gas near the space shuttle surfaces affected by the presence of the energetic fluxes of electrons and ions? This includes interaction with the surfaces, excitation of the neutrals and ionized components, and plasma etching.

Many similar questions can be formulated, but in essence they are part of two broader questions:

1. What generates the anomalous plasma environment of the shuttle?

2. What is the role of the anomalous plasma environment in the neutral gas-orbiter surface equilibrium and the associated optical emissions?

In this paper we propose that a key factor in understanding the plasma environment is collective plasma interactions of the type encountered in discharge phenomena such as Alfven critical ionization [Haerendel, 1982; Formisano et al., 1982] and beam plasma discharges [Papadopoulos, 1981]. In this context the space shuttle behaves as an artificial comet, and its glow is the equivalent of the coma. The emphasis on this preliminary and speculative note is on the basic physical processes and the expected magnitude of the phenomena, rather than the detailed modeling which should follow. In our conclusion we list a series of measurements to be performed, which will uniquely support or refute our hypothesis.

The critical ionization velocity (CIV) hypothesis invoked by Alfven [1954] in response to laboratory and cosmological observations, and based on pure free energy considerations, states that if the relative speed across the magnetic field between a plasma and a neutral gas with ionization energy $\varepsilon_{i}$ and mass $M$ exceeds a critical velocity given by

$$
U_{c}=\left(2 \varepsilon_{i} / M\right)^{1 / 2}
$$

anomalous ionization of the neutral gas by the plasma will occur. From recent laboratory [Daniel- son and Brenning, 1970] and theoretical [Raadu, 1978; Formisano et al., 1982] considerations focusing on the physics, the following picture for CIV emerged. Since the plasma cannot interact collisionlessly with the gas, some seed ionization $n_{s}$ (e.g., due to photoionization, charge exchange, etc.) is assumed, which moves with the neutral gas $N$. The orbits of newly ionized particles are such that for time scales shorter than a gyrotime $\left(\Omega_{i}^{-1}\right)$ they form a beam in velocity space (Figure $1 a$ ). The situation, thereafter, closely resembles the beam plasma discharge (BPD) [Papadopoulos, 1981], with the driver being an ion instead of an electron beam. The discharge follows the chain

seed or new ions form unstable distribution $\longrightarrow$ waves

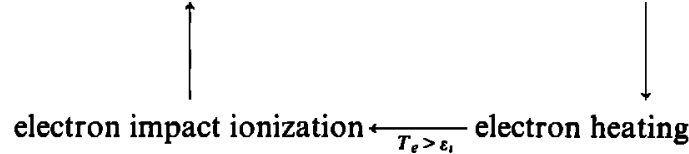

The unstable cycle continues until the electron temperature $T_{e}$ drops below $\varepsilon_{i}$, at which point the discharge is quenched. A computation of the discharge conditions can be based on phenomenological equations describing the electron energy and number
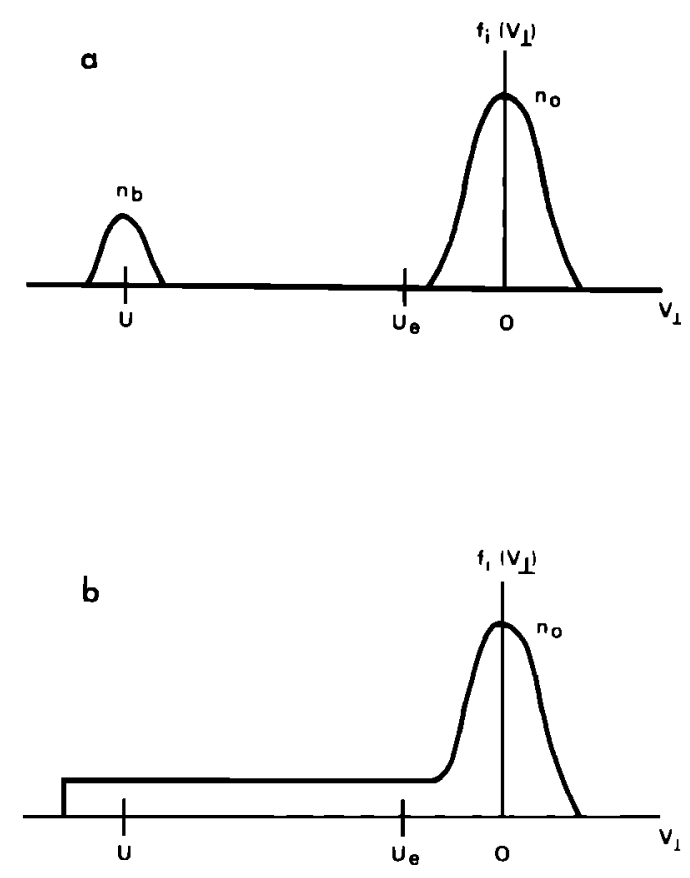

Fig. 1. Ion beam plasma velocity distribution in $V_{\perp}$ for critical ionization velocity, in the ambient ion $\left(n_{0}\right)$ reference frame. The electrons ( $n=n_{0}+n_{b}$ ) have a drift velocity $U_{e}$ to preserve zero current. (a) Initial. (b) After quasi-linear plateau is formed. 
density balance in a simple fashion [Formisano et al., 1982]:

$$
\begin{gathered}
d n T_{e} / d t=v_{\text {ion }} n\left(\eta \frac{1}{2} M U^{2}-\varepsilon_{i}\right) \\
d n / d t=v_{\text {ion }} n
\end{gathered}
$$

where $n$ is the total plasma density and $\eta$ is the fraction of ionized atom energy $\frac{1}{2} M U^{2}$ in the plasma frame transferred to electrons. From (2) we find that the minimum flow velocity to maintain a discharge $d n T_{e} / d t \geq 0$ is given by

$$
U \geq \eta^{-1 / 2} U_{c}
$$

Formisano et al. [1982] computed $\eta$ by assuming quasi-linear relaxation (Figure $1 b$ ) of the newly formed ion beam on a time scale faster than $v_{\text {ion }}^{-1}$ and $\Omega_{i}^{-1}$. In the usual electron beam quasi-linear analysis, during the plateau formation, $\frac{2}{3}$ of the beam energy remains in electrostatic waves [Davidson, 1972], since the ambient electrons are adiabatic. In the ion beam case, however, electrons can absorb the wave energy in the parallel direction, so that the resulting electron heating efficiency is $\eta=\frac{2}{3}$. Using this value, the threshold criterion becomes $U \geq 1.15 U_{c}$. We should note that these considerations assume that the electron lifetime $\tau_{L}$ in the discharge region is long with respect to the heating time $\tau_{H}$ and ionization time, i.e.,

$$
v_{\text {ion }}^{-1}+\tau_{H} \approx v_{\text {ion }}^{-1}<\tau_{L}
$$

This is equivalent to the Townsend condition, ensuring that each new heated electron has enough time to ionize.

The possibility for attributing the shuttle glow and density enhancement to CIV phenomena arises from the fact that the shuttle carries its own atmosphere which moves with the orbital velocity of $U_{0}=8 \mathrm{~km}$ $s^{-1}$ through the ambient magnetized plasma density $n_{0}$. Neutral pressures of more than $10^{-5}$ torr (i.e., $N \geq 3 \times 10^{11} \mathrm{~cm}^{-3}$ ) were measured on STS 3 [Shawhan and Murphy, 1983]. However, the value of $U_{c}$ for oxygen, assumed to be the dominant species, is $12.7 \mathrm{~km} \mathrm{~s}^{-1}$ based on an ionization energy of 13.5 $\mathrm{eV}$. The relative velocity between the plasma and the gas will be subcritical, and we will not expect CIV phenomena. In this paper we demonstrate that a more careful quasi-linear analysis and incorporation of the fact that a fraction of the plasma particles is reflected in the ram side of the orbiter allows for the possibility of CIV phenomena despite the subcritical orbital velocity.

Since the strongest effects are observed when the plasma is ramming the orbiter tail, we focus here on this case. For our purposes, it is sufficient to assume that a plasma flux equal to $\alpha n_{0} U_{0}$, where $\alpha<1$ is an unspecified reflection coefficient, streams back in the plasma after colliding with the orbiter tail. The details of the reflection and its accompanying sheath are not critical to our thesis. For time scales shorter than $\Omega_{i}^{-1}$, the velocity distribution is similar to Figure $1 a$, but with $n_{b}$ replaced by $\alpha n_{0}$ and $U=2 U_{0}$. Notice that $U=2 U_{0} \approx 16 \mathrm{~km} \mathrm{~s}^{-1}$ and is therefore larger than $U_{c}$. While we follow the quasi-linear analysis of Formisano et al. [1982] for the ion beam relaxation and retain the $\eta=\frac{2}{3}$ transfer efficiency, we also use quasi-linear theory to determine the electron distribution function. As we will see, the results indicate formation of suprathermal tails, rather than bulk electron heating with rather profound consequences. Assuming for convenience the same ionic species, the linear dispersion relation for the system of Figure $1 a$ is given by

$$
\frac{\left(n_{b} / n_{0}\right) \omega_{\mathbf{L H}}^{2}}{(\omega-\mathbf{k} \cdot \mathbf{U})^{2}}+\frac{\omega_{\mathbf{L H}}^{2}\left(1+\theta^{2}\right)}{\omega^{2}}=1
$$

where $\omega_{\mathrm{LH}}^{2}=\omega_{i}^{2} / 1+\omega_{e}^{2} / \Omega_{e}^{2}$ is the lower hybrid frequency, $\omega_{e}\left(\Omega_{e}\right)$ and $\omega_{i}\left(\Omega_{i}\right)$ are the electron and ion plasma (cyclotron) frequency, $k_{z}$ and $k_{\perp}$ are the perpendicular and parallel wavelengths, $\theta=\left(k_{z}\right)$ $k)(M / m)^{1 / 2}$, and we assumed that

$$
\begin{array}{cc}
\omega / k_{z} \gg V_{e} & \Omega_{i} \ll \omega \ll \Omega_{e} \\
k_{\perp} \approx k \ll k_{z} & k R_{e} \ll 1 \ll k R_{1} \\
|\operatorname{Im} \omega| \gg \Omega_{i} & \mathbf{B}=B_{0} \hat{e}_{z}
\end{array}
$$

( $R_{e}$ and $R_{i}$ are the electron and ion gyroradii.) For an isothermal plasma the linear analysis indicates that maximum growth occurs for $\theta=1$ and

$$
\begin{array}{ll}
\frac{\gamma(\theta)}{\gamma_{\max }}=\left(1+\theta^{2}\right)^{1 / 2} & 0 \leq \theta \leq 1 \\
\frac{\gamma(\theta)}{\gamma_{\max }}=0 & \text { elsewhere }
\end{array}
$$

The phase velocity of the unstable waves in the $z$ direction is given by

$$
V_{z}=\frac{\omega}{k_{z}}=\left(\frac{M}{m}\right)^{1 / 2} \frac{U}{\theta} \quad 0<\theta \leq 1
$$

On the basis of (7) and (8), we expect the wave spectrum to extend between $V_{1}=(M / m)^{1 / 2} U$ for $\theta=1$ and $V_{2}=2.5(M / m)^{1 / 2} U$ for $\theta=0.4$, which corre- 

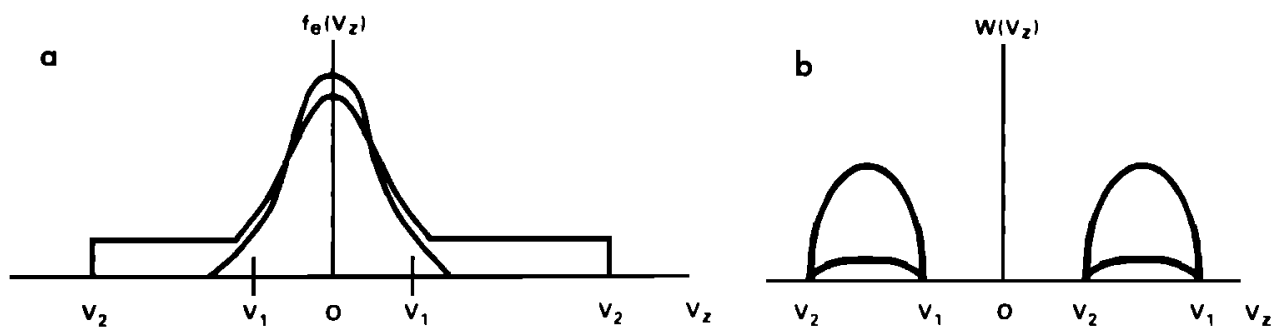

Fig. 2. Electron distribution function $f_{e}\left(V_{2}\right)$ and wave energy density $W\left(V_{z}\right)$ as a function of the parallel velocity $V_{z}$ for two times. (a) Time of quasi-linear plateau formation of the ions, which occurs before electron plateau formation. (b) Quasi-linear relaxation of electrons and wave energy.

sponds to mode energy a factor of 10 smaller than the maximum, after a few growth times.

The quasi-linear relaxation of the system (Figure $2 a$ ) is well known and results in the formation of symmetric electron tails of the form (Figure $2 b$ )

$$
\begin{array}{ll}
f_{T}=\left(V_{1}-V_{2}\right)^{-1} & V_{1} \leq V_{2} \leq V_{2} \\
f_{T}=0 & \text { elsewhere } \\
V_{1}=(M / m)^{1 / 2} U & V_{2}=2.5(M / m)^{1 / 2} U
\end{array}
$$

The number density of the tails $n_{T}$ is given by

$$
n_{T}=\frac{\frac{2}{3} n_{b} \frac{1}{2} M U^{2}}{\int_{V_{1}}^{V_{2}} f_{T} \frac{1}{2} m V_{z} d V_{z}}
$$

so that for the values given by (9),

$$
n_{T} / n_{b}=0.2
$$

From (8) we can compute the frequency of ionization for the tail particles as

$$
v_{\text {ion }}=N \int_{\left(2 \varepsilon_{1} / m\right)}^{2.5(M / m)^{1 / 2} U} f_{T}(v) v \sigma(v) d v
$$

Taking the cross section [Gurevich, 1978] as

$$
\sigma(v)=Q\left(v-\frac{\left(2 \varepsilon_{i}\right)^{1 / 2}}{m}\right)
$$

with $Q=0.5 \times 10^{-24} \mathrm{~cm} \mathrm{~s}$ for oxygen, we find from (9) and (11)

where

$$
v_{\text {ion }}=8 \times 10^{-8} N F\left(U / U_{c}\right)
$$

$$
F(x)=(x-0.4)\left(x-0.2-\frac{0.08}{x}\right)
$$

Notice that $v_{\text {ion }}$ becomes zero for $U / U_{c} \leq 0.4$, which implies that some anomalous ionization will occur for $U$ as low as $0.4 U_{c}$. Our analysis is based on a clear separation of plasma and ionization time scales, so that the distribution function of the tails and the newly born ions is a quasi-stationary plateau, while their density is time dependent (i.e., of the form $n(t) f(V))$. At this point we should also note that the value of $U$ used above is the relative streaming between electrons and the relevant ion species, which is dynamically changing to preserve zero current, i.e.,

$$
U_{e} \equiv \frac{2 \alpha+\left(n_{s} / n_{0}\right)}{1+\alpha+\left(n_{s} / n_{0}\right)} U_{0}
$$

We have three important drift velocities (Figure $3 a)$ : the relative velocity between reflected ions and electrons, $U_{r e}$, given by

$$
U_{r e}=2 U_{0} \frac{1+\frac{1}{2}\left(n_{s} / n_{0}\right)}{1+\alpha+\left(n_{s} / n_{0}\right)}
$$

the relative velocity between newly born ions and electrons, $U_{s e}$, given by

$$
U_{s e}=U_{0} \frac{1-\alpha}{1+\alpha+\left(n_{s} / n_{0}\right)}
$$

and the relative velocity between ionospheric ions and electrons, $U_{e}$, given by (13a) above.

The equation describing the density increase $n_{s}$ is

$\frac{d n_{s}}{d t} \equiv n_{0} R\left[\alpha F\left(\frac{U_{r e}}{U_{c}}\right)+\frac{n_{s}}{n_{0}} F\left(\frac{U_{s e}}{U_{c}}\right)+F\left(\frac{U_{e}}{U_{c}}\right)\right]-\frac{n_{s}}{\tau_{L}}$

where $\tau_{L}$ is the particle confinement time in the discharge region, and for our case we take it as ambipolar streaming, i.e.,

$$
\tau_{L}=L_{z} / C_{s}
$$

$L_{z}$ is the discharge dimension along the magnetic field, and $C_{s}, \approx 10^{5} \mathrm{~cm} \mathrm{~s}^{-1}$, is the sound speed. $R$ is an ionization constant based on (10) and (12) and is given by

$$
R=1.6 \times 10^{3} N_{11} \quad \mathrm{~s}^{-1}
$$

where $N_{11}=N / 10^{11} \mathrm{~cm}^{-3}$. Equations (11)-(16) form the cornerstone of our theory. It is instructive to 

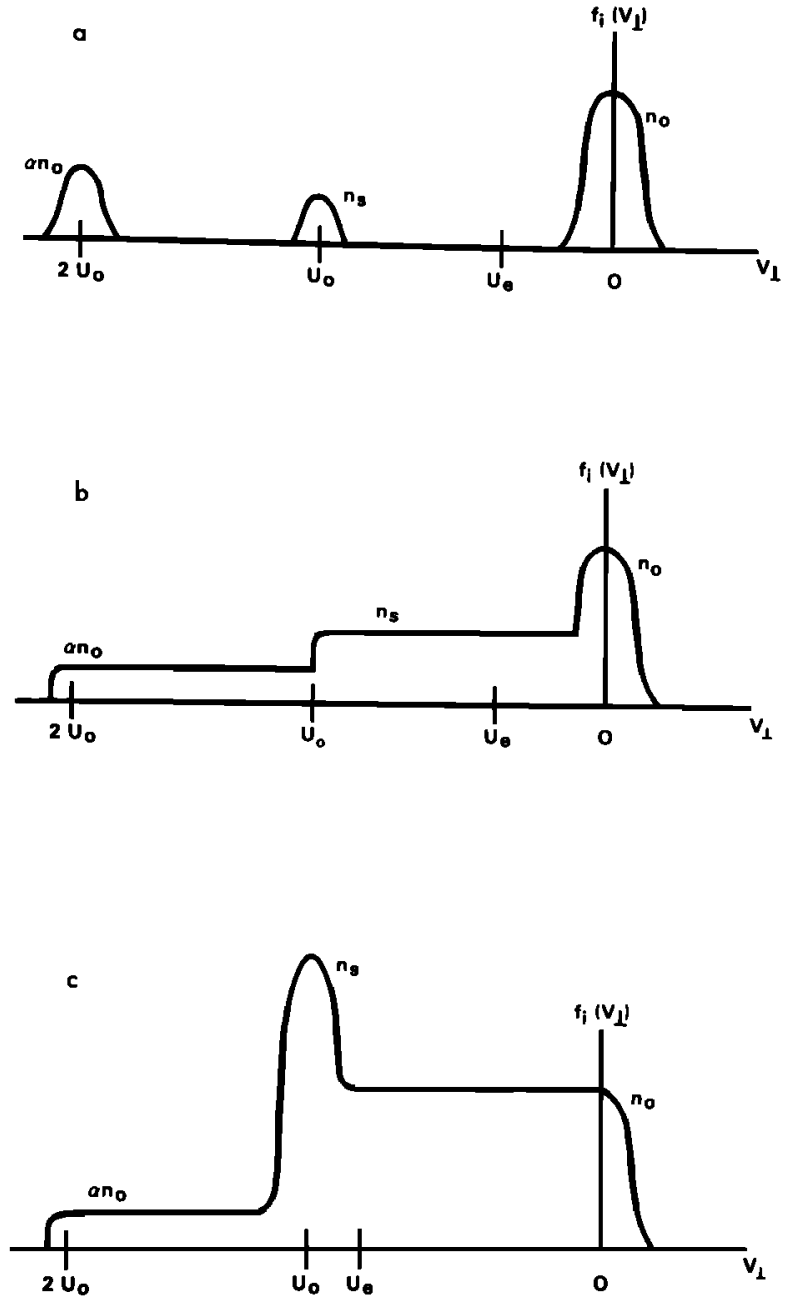

Fig. 3. Same as Figure 1, but for space shuttle case (i.e., $\alpha \neq 0$ ). (a) Initial state with $n_{0}>\alpha n_{0}>n_{s}$. (b) Quasi-linear state with $n_{0}>n_{s}>\alpha n_{0}$. (c) Asymptotic quasi-stationary case with $n_{s}>n_{0}>\alpha n_{0}$.

examine (14) more closely. Two terms, $\alpha F\left(U_{r e} / U_{c}\right)$ and $F\left(U_{e} / U_{c}\right)$, produce linear increases in density similar to BPD, while the term $\left(n_{s} / n_{0}\right) F\left(U_{s e} / U_{0}\right)$ produces an exponential increase. Initially, $U_{r e} \approx 2 U_{0}$, $U_{s e} \approx U_{0}$, and $U_{e} \approx 0$. A linear increase in the density is followed by an exponential increase if

$$
R F\left(U_{0} / U_{c}\right)>1 / \tau_{L}
$$

We will examine this condition later. The exponential phase terminates when $F\left(U_{s e} / U_{c}\right)=0$, which occurs for $U_{s e} / U_{c}=0.4$. From (13c) this occurs when $n=n_{s}+n_{0} \approx 1.3 n_{0}$. This is followed by a linear increases driven by the terms

$$
n_{0} R\left[\alpha F\left(U_{r e} / U_{c}\right)+F\left(U_{e} / U_{c}\right)\right]
$$

Notice that at this point, $U_{r e} \approx U_{e} \approx U_{0}$, while $U_{s e} \approx 0$. The steady state can be easily found as

$$
\begin{aligned}
\frac{n_{s}}{n_{0}} & =R \tau_{L} F\left(\frac{U_{0}}{U_{c}}\right)=R \frac{L_{z}}{C_{s}} F\left(\frac{U_{0}}{U_{c}}\right) \\
& =5\left(\frac{P}{10^{-5} T}\right)\left(\frac{L_{z}}{m}\right) F\left(\frac{U_{0}}{U_{c}}\right)
\end{aligned}
$$

Notice that $L_{z}$ is in units of meters. For our case, i.e., $U_{0} \approx 8 \mathrm{~km} \mathrm{~s}^{-1}, U_{c} \approx 12.7 \mathrm{~km} \mathrm{~s}^{-1}$, and $F\left(U_{0} / U_{c}\right) \approx$ 0.1 ,

$$
\frac{n_{s}}{n_{0}}=\frac{1}{2}\left(\frac{P}{10^{-5} T}\right)\left(\frac{L_{z}}{m}\right)
$$

Notice that the condition for exponential growth, given by the inequality (17), coincides with the requirement for achieving $n_{s} / n_{0}>1$. For values of $P \simeq 30-40 \mu \mathrm{T}$ and $L_{z}$ of the order of $5-10 \mathrm{~m},(18 \mathrm{~b})$ predicts density increases, near the orbiter, of a few times the ambient density, consistent with the observations.

An additional constraint imposed on the density that can be achieved comes from the free energy (equation (2)), which in our case is substantially modified. The average electron energy transfer $(d / d t) n\langle w\rangle$ will be given by

$$
\begin{aligned}
& \frac{d}{d t} n\langle w\rangle=\frac{2}{3} \alpha n_{0} \frac{\frac{1}{2} M U_{r e}^{2}}{\tau_{r}}+\frac{d n_{s}}{d t} * \frac{2}{3} \times \frac{1}{2} M U_{s e}^{2} \\
& \quad+\frac{2}{3} n_{0} \frac{\frac{1}{2} M U_{e}^{2}}{\tau_{a}}-\varepsilon_{i} \frac{d n_{s}}{d t}
\end{aligned}
$$

where $\tau_{r}$ and $\tau_{a}$ are the heating times or equivalently the relaxation times of the reflected and ambient ions respectively. They are given by their quasi-linear values, i.e.,

$$
\begin{aligned}
& \tau_{r}=\left(n / \alpha n_{0}\right) \omega_{\mathrm{LH}}^{-1} \\
& \tau_{a}=\left(n / n_{0}\right) \omega_{\mathrm{LH}}^{-1}
\end{aligned}
$$

Notice that we used $\eta=\frac{2}{3}$ in (19). In order to achieve and maintain the densities derived from (14), $(d / d t) n\langle w\rangle \geq 0$. For $\alpha=0$ and $U_{e} \approx 0$, we recover the Formisano et al. [1982] result as given by (4), as a condition for the discharge. Equation (19) allows us fo answer two important questions for the case of the orbiter environment. The first one concerns the minimum value of $\alpha$ required for discharge ignition. This can be found from (14) and (19), with $U_{r e}=2 U_{0}$, $U_{s e}=U_{0}$, and $U_{e} \approx 0$, and is given by

$$
\alpha>\frac{3}{2} \frac{\varepsilon_{i}}{\frac{1}{2} M U_{r e}^{2}} \frac{R F}{\omega_{\mathrm{LH}}} \approx 2.8 \times 10^{-2} N_{11}
$$


Namely, an $8 \%$ reflection coefficient is required for discharge ignition if $N_{11} \approx 3$. Notice that inclusion of charge exchange can reduce this requirement. The second question concerns an estimate of the energy deposition to electron tails in the discharge region and the fraction which goes to ionization, in the steady state, with $n_{s} / n_{0}>1$. In order to find concrete numbers, we take the case where $P \approx 20 \mu \mathrm{T}$ and $L_{z} \approx 3 \mathrm{~m}$, which according to $(18 b)$ results in a value of $n / n_{0}=4$. In this case the energy deposition rate to hot electrons is due to the third term in (19) and is given by

$$
Q_{0}=\frac{2}{3} n_{0} \frac{\frac{1}{2} M U_{0}^{2}}{\left(n / n_{0}\right) \omega_{\mathrm{LH}}^{-1}}
$$

For $\frac{1}{2} M U_{0}^{2} \approx 5 \mathrm{eV}, n / n_{0} \approx 4, n_{0} \simeq 10^{6} \mathrm{~cm}^{-3}$, and $\omega_{\mathrm{LH}} \approx 5 \times 10^{4},(21)$ gives

$$
Q_{0} \approx 6 \times 10^{10} \mathrm{eV} \mathrm{cm}^{-3} \mathrm{~s}^{-1}
$$

For the same parameters the energy rate spent in ionization is

$$
Q_{\text {ion }}=n_{0} R F\left(\frac{U_{0}}{U_{\mathrm{c}}}\right) \varepsilon_{i}=10^{10} \mathrm{eV} \mathrm{cm}^{-3} \mathrm{~s}^{-1}
$$

Namely, only $16 \%$ of the energy goes to ionization while most of the hot electrons (i.e., $80 \%$ ) stream out of the discharge region without making ionizing collisions, as is expected in a situation where the ionization time is comparable to or longer than the hot electron confinement time in the discharge region.

Our theoretical analysis, as given above, allows the prediction of many observations in the vicinity of the orbiter. We discuss first the stationary state based on Figure 3.

1. The density will be given by $(18 b)$. It will scale linearly with the ambient plasma density $n_{0}$, the pressure in the orbiter vicinity, and the size $L_{z}$ parallel to the surface. For typical conditions, $n \approx 3-7 n_{0}$.

2. The size of the discharge $L_{0}$ perpendicular to the surface will be given by the relaxation length

$$
L_{0}=\frac{U_{0}}{\tau_{a}} \approx \frac{n}{n_{0}} \frac{U_{0}}{\omega_{\mathrm{LH}}} \approx 3-7 \frac{U_{0}}{\omega_{L H}} \approx 10-20 \mathrm{~cm}
$$

This is fairly consistent with the observations.

3. The electron tail energy will vary between 5 and $30 \mathrm{eV}$. The tail density is given by (9), as $n_{T} /$ $n_{0}=0.2$. This will give electron fluxes in the range of $20-30 \mathrm{eV}$ of the order of $10^{11}-10^{12} \mathrm{el} \mathrm{cm}^{-2} \mathrm{eV}^{-1}$.

4. Ion temperatures in the range of $10 \mathrm{eV}$ as well as ion beams with energies in the range $5-10 \mathrm{eV}$ will be observed.
5. The spectrum of electrostatic waves excited by the interaction of the counterstreaming ions will be between

$$
f=(1-1.4) f_{\mathrm{LH}} \approx 6-9 \mathrm{kHz}
$$

with amplitudes of the order of $\mathrm{V} / \mathrm{m}$. Since in dynamic equilibrium, waves with all phase velocities $V_{\mathrm{ph}}$ in the range $-V_{0} \leq V_{\mathrm{ph}} \leq V_{0}$ are generated in the ionospheric frame, the spectrum observed from the shutthe will extend over a frequency range from nearly zero to approximately $20 \mathrm{kHz}$. Higher-frequency waves, $f_{c e}>f \gg f_{\mathrm{LH}}$, will be of the whistler type. Such waves will be produced by anisotropies during the formation of electron tails, and their signature. Finally, we expect occasional $\omega_{e}$-related emissions due to collective bremsstrahlung, which will be suppressed for high neutral densities $\left(P \geq 10^{-4} \mathrm{~T}\right)$.

6. The energetic electrons will produce line emissions characteristic of the existing neutral and ionized environment. If oxygen or $\mathrm{H}_{2} \mathrm{O}$ is the dominant species, then strong 7000 - to $8000-\AA$ emissions will be expected. However, the presence of $\mathrm{H}_{2} \mathrm{O}, \mathrm{NH}_{3}$, and $\mathrm{C}_{2} \mathrm{H}_{6}$ will produce various longer wavelengths up to IR.

7. Since the strength of interaction depends on the value of the ambient density $n_{0}$, we expect strong variations between daytime and nighttime conditions. The altitude dependence will reflect the altitude dependence of $n_{0}$.

8. The strength of the interaction depends on the value of pressure $P$. It is therefore expected that thruster operation will strongly enhance the observed glow and wave amplitudes.

The transient state will be similar to the stationary state, but with electron energies up to $100 \mathrm{eV}$, and directed ion beam energies of up to $20 \mathrm{eV}$. However, the number densities involved with be smaller by order of magnitude. The observational evidence [Shawhan and Murphy, 1983], although still rather incomplete, provides very strong support for the above predictions.

Before closing, we should stress the preliminary nature of our results. Several issues dealing with the details of the magnetic field orientation and the finite size of the ion beam in the direction parallel to the magnetic field have not been examined. These are currently under study and will be reported in the future.

In summary, we have presented a model which attributes the anomalous ionization and glow observed in the space shuttle to CIV phenomena driven 
by plasma instabilities. Our study demonstrates the feasibility of the process despite the fact that $U_{0}<$ $U_{c}$. A proper model should include, in addition to the dominant, plasma physics processes discussed above, the interplay with chemical processes. On the experimental side there is an urgent need for comprehensive simultaneous in situ measurements of the plasma parameters discussed above, the neutral gas constituency, and spectrometry from IR to UV. Active experimentation by using either thruster experimentation or neutral gas releases, such as the ones planned in SEPAC, will substantially help in elucidating the mechanism. Another set of active experiments should include the use of large plates from different materials in the bay region. which will be at various directions with respect to the direction of the velocity vector and the ambient magnetic field.

Acknowledgments. The author acknowledges several interesting discussions with A. Drobot, J. Lynch, and S. Shawhan. Many valuable suggestions and corrections on the final manuscript by $L$. Linson are gratefully acknowledged. The work was supported by SAI internal funds.

\section{REFERENCES}

Alfven, H., On the Origin of the Solar System, Oxford University Press, New York, 1954.

Banks, P. M., P. R. Williiamson, and W. J. Raitt, Space shuttle glow observations, Geophys. Res. Lett., 10, 118-121, 1983.

Danielson, L., and N. Brenning, Experiment on the interaction between a plasma and a neutral gas, Phys. Fluids, 18, 661-671, 1970.

Davidson, R. C., Methods in Non-Linear Plasma Theory, pp. 174187, Academic, New York, 1972.

Formisano, V., A. A. Galeev, and R. Z. Sagdeev, The role of critical ionization velocity phenomena in the production of inner coma cometary plasma, Planet. Space Sci., 30, 491-497, 1982.

Gurevich, A. V., Nonlinear Phenomena in the Ionosphere, p. 77, Springer-Verlag, New York, 1978.

Haerendel, G., Alfven's critical velocity effect tested in space, $Z$. Naturforsch., 379, 728-734, 1982.

Mende, S. B., O. K. Garriott, and P. M. Banks, Observations of optical emissions on STS-4, Geophys. Res. Lett., 10, 122-125, 1983.

Papadopoulos, K., The theory of the beam plasma discharge, in Artificial Particle Beams in Space, edited by B. Grandal, pp. 505-524, Plenum, New York, 1981.

Raadu, M. A., The role of electrostatic instabilities in the critical ionization velocity mechanism, Astrophys. Space Sci., 55, 125$138,1978$.

Shawhan, S., and G. Murphy, Plasma diagnostics package assessment of the STS-3 orbiter environment and system for science, Rep. AIAA-83-0253, Am. Inst. of Aeronaut. and Astronaut., New York, 1983.

Slanger, T. G., Conjectures on the origin of the surface glow of space vehicles, Geophys. Res. Lett., 10, 130-132, 1983.

Torr, M. R., Optical emissions induced by spacecraft-atmosphere interactions, Geophys. Res. Lett., 10, 114-117, 1983.

Yee, J. H., and V. J. Abreu, Visible glow induced by spacecraftenvironment interaction, Geophys. Res. Lett., 10, 126-129, 1983.

K. Papadopoulos, Department of Physics and Astronomy, University of Maryland, College Park, MD 20742. 